\title{
The multiple personalities of $\mathrm{h}$-channels
}

\author{
Bina Santoro ${ }^{1}$ and Tallie Z. Baram ${ }^{2}$ \\ ${ }^{1}$ Center for Neurobiology and Behavior, Columbia University, New York, NY 10032, USA \\ ${ }^{2}$ Anatomy, Neurobiology and Pediatrics, University of California at Irvine, Irvine, CA 92697, USA
}

\begin{abstract}
Concepts regarding the function of the hyperpolarization-activated current $\left(I_{\mathrm{h}}\right)$ in shaping the excitability of single cells and neuronal ensembles have been evolving rapidly following the recent cloning of genes that encode the underlying ' $h$-channels' - the $H C N$ genes. This article reviews new information about the transcriptional regulation of these channels, highlighting novel studies that demonstrate short- and long-term modulation of HCN expression, and linking this modulation to mechanisms of neurological diseases.
\end{abstract}

The original persona of the hyperpolarization-activated current $\left(I_{\mathrm{h}}\right)$, which was first recognized in heart, retina and motor neurons [1,2], was already multifaceted and complex. This current, originally termed $I_{\mathrm{f}}$ for 'funny', was found to perform distinct functions in different cell populations, although the most prominent role - initially proposed following work on the cardiac sinoatrial node -was generation of rhythmic depolarization [3,4]. In the proposed scenario, membrane hyperpolarization following an action potential activated $I_{\mathrm{h}}$, promoting cation entry into the cell and depolarization back towards threshold. The highly voltage-sensitive $I_{\mathrm{h}}$ de-activated concurrently with the progressive membrane depolarization, and was shut off as a new action potential was triggered. Finally, during the post-firing hyperpolarization, $I_{\mathrm{h}}$ re-activated, repeating the cycle: a pacemaker was born.

Views on the 'true personality' of $I_{\mathrm{h}}$, and the h-channels responsible for it, have been evolving ever since. The contribution of h-channels to cardiac pacemaking was soon questioned, because their slow kinetics and negative threshold of activation were deemed incompatible with the time-scale and voltage range of spontaneous electrical activity $[5,6]$. Thus, rather than driving diastolic depolarization, the primary role of $I_{\mathrm{h}}$ was proposed to be as a safety net, stabilizing membrane potential in a range where true pacemaking conductances could operate. Importantly, as the presence of h-channels was progressively recognized in neurons, analogous pacing functions in spontaneous oscillatory activity were proposed (e.g. in the thalamocortical network $[7,8]$ ). In addition, fundamental contributions to the regulation of resting potential, membrane input resistance and synaptic integration have been ascribed to $I_{\mathrm{h}}$ in neurons that are not spontaneously active, such as hippocampal CA1 and layer V cortical pyramidal neurons [9-14]. Accompanying this variety of functions, there has been a remarkable heterogeneity in the specific properties of $I_{\mathrm{h}}$ observed in different cell types with regards to kinetics, steady-state voltage dependence of activation, and sensitivity to cAMP.

More recently, an additional facet of the h-channel persona has emerged: the persistent modulation of these channels under neuropathological conditions, including denervation $[15]$ and hippocampal hyperexcitability induced by seizures [16,17]. These studies raised the 
possibility that hyperpolarization-activated currents might be subject to long-term activitydependent regulation and could play a role in hippocampal malfunction during human neurological disorders. However, the precise nature of the molecular changes in h-channels, and whether they contribute to the pathogenic process or are compensatory and neuroprotective, have been topics of intense speculation. This article highlights recent findings on the molecular basis of h-channel diversity and focuses on the dynamic regulation of h-channel expression in normal and pathological conditions, using the hippocampus as a model neuronal circuit.

\section{The HCN gene family: independent genes form distinct homomeric channels}

Recent cloning of the hyperpolarization-activated cyclic nucleotide-gated ( $\mathrm{HCN})$ gene family has provided a structural basis for the observed heterogeneity of native $I_{\mathrm{h}}$. Each of the four characterized mammalian $\mathrm{HCN}$ genes $(\mathrm{HCN} 1-\mathrm{HCN} 4)$ [18] generates currents with the general properties of $I_{\mathrm{h}}$ when expressed heterologously, including activation upon hyperpolarizing voltage steps below $-60 \mathrm{mV}$ and positive modulation by binding of cAMP. However, the four homomeric $\mathrm{HCN}$ channels differ considerably in their intrinsic biophysical properties -namely, in their kinetics of activation and deactivation (which are fastest for HCN1 and slowest for HCN4) and in their cAMP-evoked shift in voltage dependence.

The expression patterns of the four $\mathrm{HCN}$ genes in brain are also quite distinct: $\mathrm{HCNl}$ is found mainly in cortical regions, whereas $H C N 4$ is expressed almost exclusively in subcortical regions $[19,20]$. $H C N 2$ and $H C N 3$ are expressed in a wider range of discrete neuronal populations, although the level of expression of the latter is low. In general, mRNA expression profiles of HCN genes have correlated reasonably well with the properties of $I_{\mathrm{h}}$ recorded from individual neurons $[20,21]$. For example, $I_{\mathrm{h}}$ recorded from thalamocortical relay neurons, which express $\mathrm{HCN} 2$ and $\mathrm{HCN} 4$, have much slower kinetics and higher cAMP sensitivity than $I_{\mathrm{h}}$ recorded from hippocampal CA1 pyramidal neurons, which express high levels of HCN1 and HCN2 [17,20]. Although there are exceptions, this correlation suggests that cell-specific differences in the biophysical properties of $I_{\mathrm{h}}$ are, in large part, determined on a transcriptional level.

Finally, differential regulation of $\mathrm{HCN}$ isoform expression has been described during CNS development $[22,23]$. Detailed studies of the spatiotemporal expression patterns of HCN genes in hippocampus have revealed important differences in $\mathrm{HCN} 1$ and $\mathrm{HCN} 2$ expression within distinct neuronal subpopulations, as well as in the temporal progression of this expression. Thus, expression in principal cells precedes that in interneurons for both isoforms [22]. Furthermore, within interneuron populations, the progressive expression of $\mathrm{HCN} 1$ is confined primarily to parvalbumin-expressing basket-type cells, whereas HCN2 is more prominent in other interneuron populations. Within pyramidal cells, several dynamic changes in the expression of $\mathrm{HCN} 1$ and $\mathrm{HCN} 2$ subunits, and in the corresponding density and kinetics of $I_{\mathrm{h}}$, have been observed during the first three postnatal weeks [22,23].

\section{Activity-dependent regulation of $\mathbf{h}$-channel expression}

Although the mechanisms underlying regulation of $\mathrm{HCN}$ gene expression during development remain unresolved, it is tempting to speculate that the evolving network activity of the hippocampal circuit might govern differential HCN expression. Direct evidence for an effect of activity on h-channel expression has been obtained using rodent models of human childhood seizures and epileptogenesis. Hyperthermia-induced seizures in immature (postnatal-day ten) rats induce long-lasting alterations in limbic neuronal 
excitability, including enhancement of inhibitory postsynaptic currents (IPSC) and chronic enhancement of $I_{\mathrm{h}}$ in CA1 pyramidal neurons [16]. Seizure activity in this developmental model resulted in a small but crucial depolarizing shift of the voltage dependence of $I_{\mathrm{h}}$, as well as a significant slowing in its activation and deactivation kinetics. These changes were persistent and, in combination with the increased perisomatic IPSCs, led to increased rebound firing of CA1 neurons and a hyper-excitable hippocampal network. On a molecular level, prolonged seizures (induced by kainic acid as well as hyperthermia) in the immature rat exerted a dose-dependent and differential effect on HCN1 and HCN2 transcription [17]. Levels of HCN2 mRNA were significantly and persistently increased in CA1 and CA3 hippocampal pyramidal cell layers, whereas a concurrent reduction of HCN1 was confined to CA1. These data demonstrate both isoform and regional specificity in the modulation of HCN gene expression by intense network activity. Importantly, the increased ratio of HCN2 versus $\mathrm{HCN} 1$ is consistent with the observed slowing of the activation and deactivation kinetics of $I_{\mathrm{h}}$ in CA1 neurons. It also persisted for weeks, further suggesting that the longlasting changes in h-channel properties might be, at least in part, sustained on a transcriptional level.

The above findings not only highlight a dynamic regulation of h-channel expression in the hippocampus in vivo but also indicate that the predominant isoforms expressed in this region, $\mathrm{HCN} 1$ and $\mathrm{HCN} 2$, can be differentially regulated in response to activity. Independent regulation of $\mathrm{HCN} 1$ and $\mathrm{HCN} 2$ expression has also been reported after lesioninduced neuronal plasticity in adult hippocampus [15]. In this model, transection of the perforant path and resulting partial denervation of dentate gyrus granule cells and CA1 pyramidal cells resulted in a strong downregulation of HCN1 without change in the expression of other $\mathrm{HCN}$ isoforms. Compelling evidence for the differential regulation of $\mathrm{HCN}$ isoforms in human neuropathological conditions has been provided by analysis of $\mathrm{HCN} 1$ and $\mathrm{HCN} 2$ expression in human epileptic hippocampus. Although there was little alteration of $\mathrm{HCN} 2$ expression, a striking increase of HCN1 mRNA was evident in the injured dentate gyrus, within surviving granule cells. Importantly, HCN1 immunoreactivity was markedly increased in the dendritic compartments of granule cells that were bombarded by dendritic excitation from abnormal, sprouted axons (mossy fibres) of neighboring surviving granule cells [24].

The protracted time-course of the changes in HCN expression is consistent with the notion that they are determined on a transcriptional level. However, acute activity-dependent upregulation of $I_{\mathrm{h}}$ in vitro, in the hippocampal slice preparation, was also recently shown $[25,26]$. Although details of the properties and mechanisms underlying this modulation are not yet available, these topics are expected to be the subject of intense and exciting future research.

\section{The role of normal and modified $\mathrm{h}$-channels in neuronal excitability: unresolved conflicts or a split personality?}

Taken together, recent and ongoing studies demonstrate that neuronal activity, both normal and aberrant, might be a major determinant of $\mathrm{HCN}$ expression. In turn, the resulting novel array of $\mathrm{HCN}$ channel properties can potently influence the excitability of the hippocampal network. But what are the specific consequences of altered $I_{\mathrm{h}}$ density, gating properties or molecular composition on neuronal and network excitability?

Using both genetic and activity-dependent model systems, some studies support an inverse correlation between $I_{\mathrm{h}}$ amplitude and seizure threshold. Thus, the depolarizing shift leading to an enhanced hyperpolarization-activated conductance in CA1 pyramidal cells is key to the hyperexcitable hippocampus in the febrile seizure model [16]. In addition, increased 
maximal $I_{\mathrm{h}}$ is found in cortical layer $\mathrm{V}$ pyramidal cells of the stargazer mouse, a single-locus mutant with a phenotype of spike-wave seizures [27]. Although a causal relationship between $I_{\mathrm{h}}$ and spontaneous seizures has not been established in this model, it has been suggested that $I_{\mathrm{h}}$ in cortical pyramidal neurons might be involved in the generation of postinhibitory excitation leading to oscillatory paroxysmal activity [28].

By contrast, a neuron-stabilizing and neuroprotective effect of $I_{\mathrm{h}}$ has been suggested by a study in which $I_{\mathrm{h}}$ was enhanced pharmacologically [29]. When a large depolarizing shift in $\mathrm{h}$-channel activation $(\sim 11 \mathrm{mV})$ was induced by the anticonvulsant drug lamotrigine in CA1 pyramidal cells, repetitive action potential firing elicited by dendritic (but not somatic) current injections was significantly reduced. The proposed mechanism is that the lamotrigine-induced increase in the steady-state activation of $I_{\mathrm{h}}$ at potentials near rest reduces membrane input resistance and time constant, resulting in reduced temporal summation and shunting of dendritic excitatory synaptic inputs $[9,10,29]$. This interpretation is consistent with the suggested neuroprotective and compensatory role for the increased HCN1 expression observed in the injured, depleted dentate gyrus of the human hippocampus [24]. The striking increase of HCN1 mRNA levels and concomitant increase in protein expression in the granule cell dendritic field would constitute a compensatory attempt to limit abnormal hyperexcitability in the epileptic dentate gyrus.

These seemingly conflicting facets of the $I_{\mathrm{h}}$ persona can be reconciled by considering that both intrinsic and extrinsic factors are likely to play a role in determining the net impact of a given change in $\mathrm{HCN}$ function on neuronal and network excitability. These include other ionic conductances present in the cell (i.e. the 'electrophysiological environment' in which the HCN channels operate), the nature and spatial distribution of synaptic inputs, and the specific molecular composition, biophysical properties and subcellular localization of the hchannels that are involved.

Thus, although the enhanced steady-state activation of $I_{\mathrm{h}}$ in both the febrile seizures model and following lamotrigine application will decrease input resistance in CA1 pyramidal neurons (hence the magnitude of voltage deflections in response to current inputs), the nature and the location of synaptic input onto the CA1 neurons differ in these two scenarios (Figure 1). A concurrent increase in hyperpolarizing perisomatic inhibitory input to CA1 neurons is found in the developmental seizure model; this will activate $\mathrm{h}$-channels and drive rebound depolarization and firing, leading to a hyperexcitable hippocampal network. By contrast, enhancement of $I_{\mathrm{h}}$ by lamotrigine dampens repetitive firing elicited by dendritic (but not somatic) depolarization. The uneven distribution of ionic conductances that operate in the subthreshold range (e.g. $I_{\mathrm{h}}$ itself, as well as the transient potassium current $I_{\mathrm{A}}$ and the low-voltage-activated $\mathrm{Ca}^{2+}$ current $I_{\mathrm{T}}$ [30]) in CA1 pyramidal neurons is likely to account for important differences in the neuronal response to localized synaptic inputs. Moreover, ratios among different currents, rather than absolute expression levels, are likely to determine the nature of the electrical response. For example, the interaction between $I_{\mathrm{h}}$ and $I_{\mathrm{A}}$ sets the rate of post-inhibitory rebound. However, the relative expression levels of these two conductances are kept in tight homeostatic equilibrium in invertebrate pacemaker neurons [31]. Overexpression of Kv4-type channel proteins in this model leads to a compensatory upregulation of $I_{\mathrm{h}}$ density, with little net change in firing properties of the neuron. If such compensatory mechanisms exist in neurons, absent or inappropriate compensatory changes between functionally linked ionic conductances might lead to an altered electrophysiological environment and altered neuronal responses, with disruption of normal network excitability.

Activity-induced changes in the molecular subunit composition of h-channels will also influence their gating properties, including rate of activation and deactivation. Indeed, 
concurrent upregulation of $\mathrm{HCN} 2$ and down-regulation of HCN1 mRNA expression were found in developmental seizures models [17]. The resulting altered gating properties of the cellular complement of h-channels might lead to different neuronal responses and network excitability. In addition, a change in molecular subunit composition might alter the localization of the h-channels.

Thus, individual $\mathrm{HCN}$ isoforms might be targeted to different subcellular compartments. Immunocytochemical analyses of HCN1 distribution have demonstrated a marked segregation of this subunit to the apical dendrites of hippocampal pyramidal cells $[17,32,33]$. The progressive enhancement of $\mathrm{HCN} 1$ density with increasing distance from the soma is consistent with the somatodendritic gradient of $I_{\mathrm{h}}$ density $[9,10]$. However, a similar gradient was not observed upon analysis of HCN2 distribution [17,23]. Finally, channels composed of different $\mathrm{HCN}$ isoforms might differ in their interaction with their cellular environment. For example, they might be linked to unique ensembles of receptors, other channels or regulatory proteins within the same cell. As a result, the relative expression levels of specific HCN subunits might ultimately determine the influence of $I_{\mathrm{h}}$ on neuronal electrophysiological properties.

\section{Do HCN proteins form heteromeric channels?}

Despite the diverse spatial and temporal expression patterns of HCN genes in the CNS, substantial overlap is found within several regions, and multiple isoforms can be coexpressed in a single neuron [17,21]. Several recent studies have also provided strong evidence for the formation of heteromeric channels in heterologous systems [14]. However, whether heteromeric $\mathrm{HCN}$ channels generate native neuronal $I_{\mathrm{h}}$ has not been definitively resolved. In theory, HCN subunits might assemble in vivo as homomeric channels, as heteromers, or both. Indeed, abundance-dependent formation of homomeric or heteromeric channels has been described for voltage-gated $\mathrm{K}^{+}$channels, specifically Kv1.5. This subunit produces both homomeric and heteromeric channels (with Kv1.4), depending on expression levels [34]. Thus, it could be that the relative expression (i.e. the relative abundance) of $\mathrm{HCN}$ isoforms might govern their assembly. This is particularly intriguing in view of the recent work indicating that $I_{\mathrm{h}}$ with novel properties might arise in vivo in hippocampal neurons, under pathological conditions that also alter the relative abundance of the HCN1 and $\mathrm{HCN} 2$ isoforms.

\section{Multiple personalities of $\mathbf{h}$-channels: past and future}

The delineation of discrete $\mathrm{HCN}$ genes has provided the conceptual foundation for channels with independent locations, regulation and physiological properties. Can we speculate about the evolutionary basis of this diversity, and predict future advantageous use of this diversity?

The multiplicity of HCN genes might provide a safety mechanism, whereby a high degree of redundancy guarantees function of a crucial regulator of intrinsic cellular excitability. In addition, because homomeric $\mathrm{HCN}$ gene products possess different gating properties, gene duplication permits biophysical fine tuning, generating specific HCN subunits that are fundamentally more suitable for distinct physiological functions. Finally, as highlighted in this review, the presence of multiple $\mathrm{HCN}$ genes provides specificity in intercellular or intracellular localization and regulation modules.

Indeed, the distinct expression patterns and differential regulation already observed for the $\mathrm{HCN}$ genes, combined with their emerging disparate associations with subcellular compartments, receptors and other channels, suggest that specific roles in modulating neuronal excitability might eventually be defined for each isoform (at least within a given network). For example, a powerful demonstration of the role of $\mathrm{HCN} 2$ in shaping the firing 
mode of thalamocortical relay neurons has been recently provided by analysis of mice carrying a null mutation in the HCN2 locus [35]. In particular, the spontaneous absence seizure phenotype of these mice highlights the interplay between $I_{\mathrm{h}}$ and T-type $\mathrm{Ca}^{2+}$ channels as a key determinant in modulating the synchronized oscillatory activity of the thalamocortical network. The upcoming characterization of mice harboring null mutations for $H C N 1$, which is prominently expressed in pyramidal cells, and of mice with hippocampus-specific deletions of $H C N 2$, will undoubtedly provide equally compelling insights into the role of these genes in the normal and aberrant function of the hippocampal network.

In conclusion, can we ascribe different functions to the different HCN isoforms, and could this be the source of some of the conflicting 'personality traits' in $I_{\mathrm{h}}$ behavior? Although a definitive answer to this question is not yet available, the concept provides an important theoretical framework for devising selective therapeutic intervention strategies and for defining specific contributions of h-channel activity to the aetiology (and treatment) of neurological disorders.

\section{Acknowledgments}

We thank E. Kandel, S. Siegelbaum and R. Bender for their comments and discussions. Our research is supported by NIH grants NS 36658 (B.S.) and NS 35439 and 28912 (T.Z.B.).

\section{References}

1. DiFrancesco D. Pacemaker mechanisms in cardiac tissue. Annu Rev Physiol 1993;55:455-472. [PubMed: 7682045]

2. Pape HC. Queer current and pacemaker: the hyperpolarization-activated cation current in neurons. Annu Rev Physiol 1996;58:299-327. [PubMed: 8815797]

3. Brown HF, et al. How does adrenaline accelerate the heart? Nature 1979;280:235-236. [PubMed: 450140]

4. Brown H, DiFrancesco D. Voltage-clamp investigations of membrane currents underlying pacemaker activity in rabbit sinoatrial node. J Physiol 1980;308:331-351. [PubMed: 7230019]

5. Yanagihara K, Irisawa H. Potassium current during the pacemaker depolarization in rabbit sinoatrial node cell. Pflugers Arch 1980;388:255-260. [PubMed: 7193851]

6. Noma A, et al. Does the 'pacemaker current' generate the diastolic depolarization in the rabbit SA node cells? Pflugers Arch 1983;397:190-194. [PubMed: 6878006]

7. Pape HC, McCormick DA. Noradrenaline and serotonin selectively modulate thalamic burst firing by enhancing a hyperpolarization-activated cation current. Nature 1989;340:715-718. [PubMed: 2475782]

8. McCormick DA, Pape HC. Properties of a hyperpolarization-activated cation current and its role in rhythmic oscillation in thalamic relay neurones. J Physiol 1990;431:291-318. [PubMed: 1712843]

9. Magee JC. Dendritic hyperpolarization-activated currents modify the integrative properties of hippocampal CA1 pyramidal neurons. J Neurosci 1998;18:7613-7624. [PubMed: 9742133]

10. Magee JC. Dendritic $I_{\mathrm{h}}$ normalizes temporal summation in hippocampal CA1 neurons. Nat Neurosci 1999;2:848. [PubMed: 10461231]

11. Williams SR, Stuart GJ. Site independence of EPSP time course is mediated by dendritic $I_{\mathrm{h}}$ in neocortical pyramidal neurons. J Neurophysiol 2000;83:3177-3182. [PubMed: 10805715]

12. Berger $\mathrm{T}$, et al. High $I_{\mathrm{h}}$ channel density in the distal apical dendrite of layer $\mathrm{V}$ pyramidal cells increases bidirectional attenuation of EPSPs. J Neurophysiol 2001;85:855-868. [PubMed: 11160518]

13. Lupica CR, et al. Contribution of the hyperpolarization-activated current $\left(I_{\mathrm{h}}\right)$ to membrane potential and GABA release in hippocampal interneurons. J Neurophysiol 2001;86:261-268. [PubMed: 11431507] 
14. Robinson RB, Siegelbaum SA. Hyperpolarization-activated cation currents: from molecules to physiological function. Annu Rev Physiol 2003;65:453-480. [PubMed: 12471170]

15. Brauer AU, et al. Molecular and functional analysis of hyperpolarization-activated pacemaker channels in the hippocampus after entorhinal cortex lesion. FASEB J 2001;15:2689-2701. [PubMed: 11726545]

16. Chen K, et al. Persistently modified h-channels after complex febrile seizures convert the seizureinduced enhancement of inhibition to hyperexcitability. Nat Med 2001;7:331-337. [PubMed: 11231632]

17. Brewster A, et al. Developmental febrile seizures modulate hippocampal gene expression of hyperpolarization-activated channels in an isoform- and cell-specific manner. J Neurosci 2002;22:4591-4599. [PubMed: 12040066]

18. Clapham DE. Not so funny anymore: pacing channels are cloned. Neuron 1998;21:5-7. [PubMed: 9697846]

19. Moosmang S, et al. Differential distribution of four hyperpolarization-activated cation channels in mouse brain. Biol Chem 1999;380:975-980. [PubMed: 10494850]

20. Santoro B, et al. Molecular and functional heterogeneity of hyperpolarization-activated pacemaker channels in the mouse CNS. J Neurosci 2000;20:5264-5275. [PubMed: 10884310]

21. Franz O, et al. Single-cell mRNA expression of HCN1 correlates with a fast gating phenotype of hyperpolarization-activated cyclic nucleotide-gated ion channels (IH) in central neurons. Eur J Neurosci 2000;12:2685-2693. [PubMed: 10971612]

22. Bender RA, et al. Differential and age-dependent expression of hyperpolarization-activated, cyclic nucleotide-gated cation channel isoforms 1-4 suggests evolving roles in the developing rat hippocampus. Neuroscience 2001;106:689-698. [PubMed: 11682156]

23. Vasilyev DV, Barish ME. Postnatal development of the hyperpolarization-activated excitatory current $I_{\mathrm{h}}$ in mouse hippocampal pyramidal neurons. J Neurosci 2002;22:8992-9004. [PubMed: 12388606]

24. Bender RA, et al. Enhanced expression of a specific hyperpolarization-activated cyclic nucleotidegated cation channel $(\mathrm{HCN})$ in surviving dentate gyrus granule cells of human and experimental epileptic hippocampus. J Neurosci 2003;23:6826-6836. [PubMed: 12890777]

25. van Welie, I., et al. Program No. 344.1, 2002 Abstract Viewer and Itinerary Planner. Society for Neuroscience; 2002. Rapid modulation of somatic hyperpolarization-activated inward currents by synaptic activity.

26. Wang Z, et al. Bidirectional changes in spatial dendritic integration accompanying long-term synaptic modifications. Neuron 2003;37:463-472. [PubMed: 12575953]

27. di Pasquale E, et al. Increased excitability and inward rectification in layer V cortical pyramidal neurons in the epileptic mutant mouse Stargazer. J Neurophysiol 1997;77:621-631. [PubMed: 9065835]

28. Timofeev I, et al. Cortical hyperpolarization-activated depolarizing current takes part in the generation of focal paroxysmal activities. Proc Natl Acad Sci U S A 2002;99:9533-9537. [PubMed: 12089324]

29. Poolos NP, et al. Pharmacological upregulation of h-channels reduces the excitability of pyramidal neuron dendrites. Nat Neurosci 2002;5:767-774. [PubMed: 12118259]

30. Migliore M, Shepherd GM. Emerging rules for the distributions of active dendritic conductances. Nat Rev Neurosci 2002;3:362-370. [PubMed: 11988775]

31. MacLean JN, et al. Activity-independent homeostasis in rhythmically active neurons. Neuron 2003;37:109-120. [PubMed: 12526777]

32. Santoro B, et al. Interactive cloning with the SH3 domain of $\mathrm{N}$-src identifies a new brain specific ion channel protein, with homology to eag and cyclic nucleotide-gated channels. Proc Natl Acad Sci U S A 1997;94:14815-14820. [PubMed: 9405696]

33. Lorincz A, et al. Polarized and compartment-dependent distribution of HCN1 in pyramidal cell dendrites. Nat Neurosci 2002;5:1185-1193. [PubMed: 12389030]

34. Levitan ES, Takimoto K. Dynamic regulation of $\mathrm{K}^{+}$channel gene expression in differentiated cells. J Neurobiol 1998;37:60-68. [PubMed: 9777732] 
35. Ludwig A, et al. Absence epilepsy and sinus dysrhythmia in mice lacking the pacemaker channel HCN2. EMBO J 2003;22:216-224. [PubMed: 12514127] 


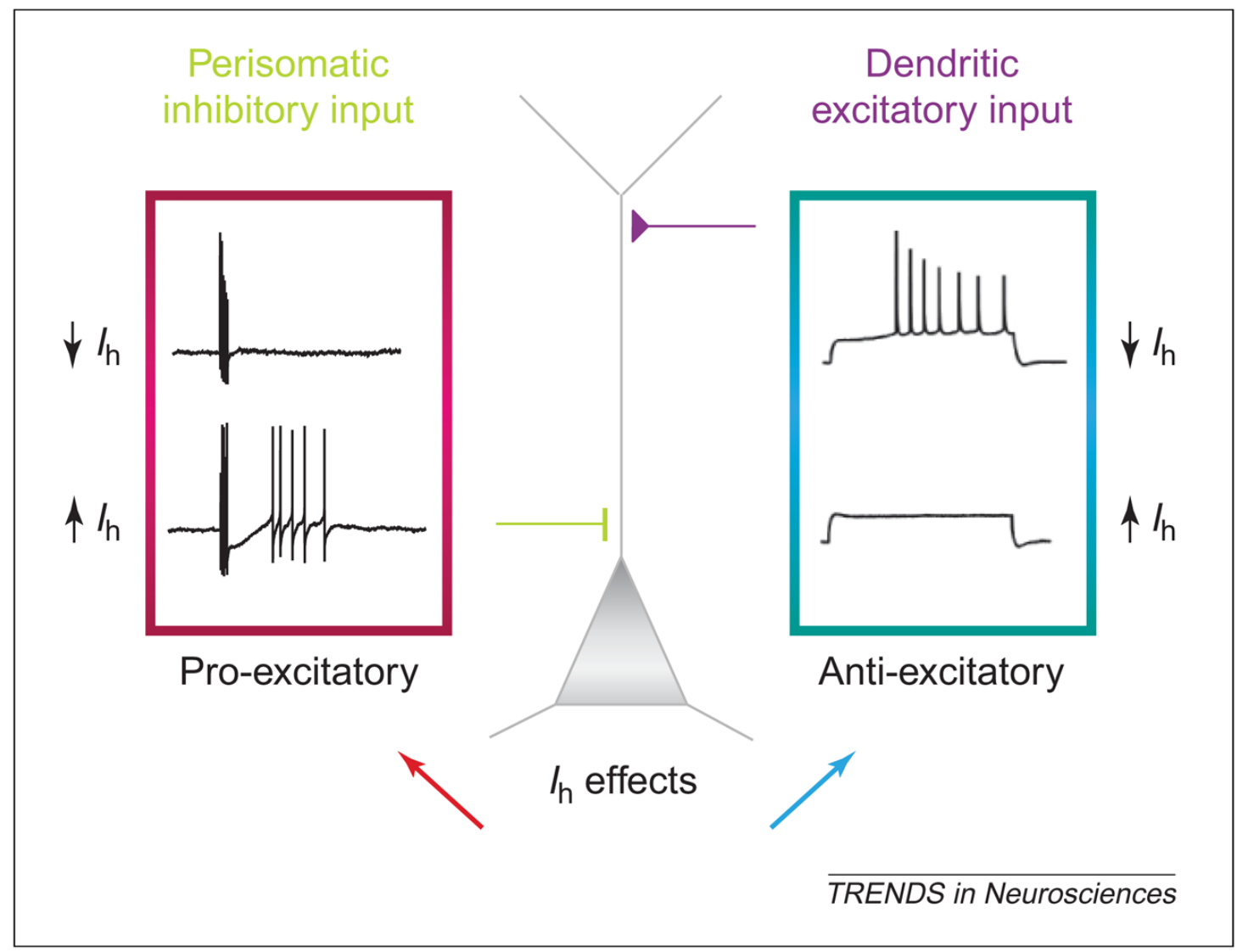

Figure 1.

Pro-excitatory or anti-excitatory? Opposing effects of increased h-channel activity on the modulation of cellular excitability have been suggested. The seemingly opposing actions can be reconciled by considering several factors. These include the spatial distribution and the nature of synaptic inputs and other intrinsic conductances present in the neuron (i.e. the electrophysiological context in which the h-channels operate), as well as the functional properties of the specific h-channel isoforms involved. Traces in the frame on the left are reproduced, with permission, from [16], (C) (2001) Nature Publishing Group (http://www.nature.com/); traces in the frame on the right are reproduced, with permission, from Ref. [29], (C) (2002) Nature Publishing Group. 